\title{
Diabetic macroangiopathy
}

\section{Quantitative histopathological studies of the extramural coronary arteries from Type 2 (non-insulin-dependent) diabetic patients}

\author{
H. Dybdahl and T. Ledet \\ University Institute of Pathology, Aarhus Kommunehospital, and Institute of Experimental Clinical Research, \\ Aarhus, University, Aarhus, Denmark
}

\begin{abstract}
Summary. A morphometric study was performed on histological sections of the extramural coronary arteries from 10 Type 2 (non-insulin-dependent) diabetic patients and 10 nondiabetic subjects, matched for age and sex. Standardised samples were taken from the two coronary arteries and the fractional contents of Periodic Acid-Schiff-positive material, acid mucopolysaccharides and connective tissue was determined in the arterial tunica media from the two groups using a point-counting technique. The thickness of tunica intima and tunica media was obtained by micrometric measurements. There was no difference in the thickness of tunica intima with and without atherosclerotic plaque between the vessels from diabetic and nondiabetic hearts. However, the thickness of tunica media was significantly reduced in arteries from the diabetic patients $(2 p<0.01)$. In tunica media of the diabetic patients there was a significant increase $(50 \%)$ in
\end{abstract}

the amount of Periodic Acid-Schiff-positive material $(2 p<$ 0.01 ), whereas the content of acid mucopolysaccharides (alcian-blue positive) was significantly reduced $(2 p<0.02)$. These changes in tunica media were similar in areas below and outside the intimal atherosclerotic plaques. The content of connective tissue was found to be significantly increased in the arteries from the diabetic patients, but only in areas outside atherosclerotic plaques $(2 p<0.025)$. There was no correlation between the observed changes in tunica media and the known duration of diabetes. The results obtained in the present study may be ascribed to the presence of a nonatherosclerotic diabetic macroangiopathy.

Key words: Macroangiopathy, diabetes mellitus, morphometry, histopathology, extramural coronary arteries.
It has been known for many years that diabetes mellitus increases the frequency of abnormalities in the arteries from the heart and legs [1]. This large vessel disease has usually been regarded as a particularly severe form of atherosclerosis. As a matter of fact, however, several studies have suggested that diabetic patients may have a macroangiopathy in addition to atherosclerosis. Recent reviews have dealt with that particular problem [2].

It is confusing that diabetic macroangiopathy has been used as a denominator for an early and severe atherosclerosis. In fact the term was introduced in the early 1970 s to emphasise the idea of a non-atherosclerotic large vessel disease in diabetic patients [3].

Information about the morphological alterations in the diabetic large vessels have mainly been obtained in studies carried out within the framework of one or another variant of the lipid-atherosclerosis hypothesis, and have focussed on the macroscopical abnormalities. Indeed, there is very little data in the area of histopathology about the alterations in large vessels from hearts obtained from diabetic patients.
The present histological investigation has analysed with morphometric techniques the coronary arteries from Type 2 (non-insulin-dependent) diabetic patients. The data obtained suggest the presence of abnormalities which may be ascribed to a non-atherosclerotic diabetic large vessel disease.

\section{Materials and methods}

The hearts of 10 Type 2 diabetic patients aged 55-80 years with a known duration of diabetes from 2-25 (mean 8.5) years were collected randomly at autopsy. The material comprises 3 females and 7 males (Table 1). Five of these died from ischaemic heart disease and the remaining from other causes (Table 2). Only 1 patient suffered from diabetic retinopathy and 4 had reduced renal function judging from the level of se-creatinine $(>150 \mu \mathrm{mol} / \mathrm{l})$. Those hearts were compared with 10 hearts from non-diabetic subjects, 3 females and 7 males aged 55-78 years. Among these, 4 died from ischaemic heart disease and 1 had reduced renal function. Excluded from both groups were patients with malignant disease, hypertension requiring treatment, hyperlipidaemia, chronic alcoholism or chronic obstructive lung disease and long-term steroid treatment. The coronary atherosclerosis was graded semiquantitatively: in grade 1 atherosclerosis there were scattered plaques without stenosis, in grade 2 
Table 1 Data of diabetic patients (no.1-10) and non-diabetic subjects (no. 11-20)

\begin{tabular}{|c|c|c|c|c|c|c|c|}
\hline & $\begin{array}{l}\text { Age } \\
\text { (years) }\end{array}$ & Sex & $\begin{array}{l}\text { Body } \\
\text { mass } \\
\text { index } \\
\left(\mathrm{kg} / \mathrm{m}^{2}\right)\end{array}$ & $\begin{array}{l}\text { Blood } \\
\text { pressure } \\
\text { (mm Hg) }\end{array}$ & $\begin{array}{l}\text { Se-creatinine } \\
(\mathrm{mmol} / \mathrm{l})\end{array}$ & Proteinuria & $\begin{array}{l}\text { Known } \\
\text { duration } \\
\text { of diabetes } \\
\text { (years) }\end{array}$ \\
\hline 1 & 55 & $\mathrm{~F}$ & 20.7 & $160 / 90$ & 400 & + & 5 \\
\hline 2 & 71 & $\mathrm{~F}$ & & $140 / 90$ & 92 & 0 & 3 \\
\hline 3 & 63 & $\mathbf{M}$ & 20.9 & $170 / 100$ & 450 & + & 8 \\
\hline 4 & 66 & M & & $160 / 90$ & 150 & 0 & 4 \\
\hline 5 & 77 & M & 22.7 & $145 / 95$ & 110 & 0 & 25 \\
\hline 6 & 65 & $\mathrm{M}$ & 23.1 & $180 / 95$ & 570 & + & 18 \\
\hline 7 & 69 & $\mathrm{M}$ & 25.9 & $150 / 90$ & 80 & 0 & 5 \\
\hline 8 & 74 & M & 28.0 & & 99 & 0 & 2 \\
\hline 9 & 80 & $\mathrm{~F}$ & 22.3 & $165 / 85$ & 113 & 0 & 11 \\
\hline 10 & 72 & $\begin{array}{l}\mathrm{M} \\
\text { mean }\end{array}$ & $\frac{25.3}{23.6}$ & $140 / 80$ & 110 & 0 & 4 \\
\hline 11 & 55 & $\mathrm{~F}$ & 24.6 & & & & \\
\hline 12 & 67 & $\mathrm{~F}$ & 21.5 & $160 / 80$ & 93 & 0 & \\
\hline 13 & 60 & $\mathrm{M}$ & 24.7 & & & & \\
\hline 14 & 68 & M & 24.1 & & & & \\
\hline 15 & 78 & $\mathbf{M}$ & & $130 / 80$ & 230 & 0 & \\
\hline 16 & 68 & $\mathbf{M}$ & 34.2 & & & & \\
\hline 17 & 68 & M & 27.3 & $170 / 70$ & 85 & & \\
\hline 18 & 72 & $F$ & 23.5 & $160 / 80$ & 81 & 0 & \\
\hline 19 & 70 & $\mathrm{M}$ & 15.1 & $180 / 100$ & 105 & 0 & \\
\hline 20 & 74 & $\begin{array}{l}\mathbf{M} \\
\text { mean }\end{array}$ & $\frac{22.6}{24.1}$ & $130 / 80$ & 102 & 0 & \\
\hline
\end{tabular}

Table 2 Causes of death among diabetic patients and non-diabetic subjects

\begin{tabular}{lll}
\hline & $\begin{array}{l}\text { Diabetic } \\
\text { patients }\end{array}$ & $\begin{array}{l}\text { Non-diabetic } \\
\text { subjects }\end{array}$ \\
\hline Cardiac insufficiency/ & 5 & 4 \\
$\quad$ coronary thrombosis & 3 & \\
Uraemia & 1 & \\
Pneumonia & 1 & 1 \\
Pulmonary embolia & & 2 \\
Ruptured aortic aneurysm & & 3 \\
Accidents & & \\
\hline
\end{tabular}

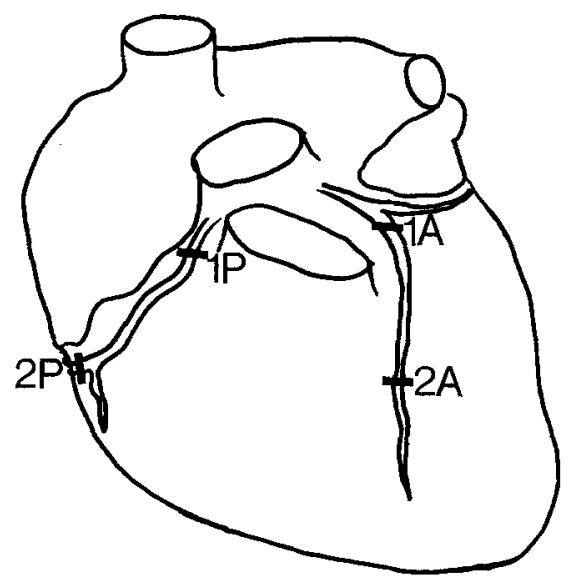

Fig. 1. Location of the arterial samples. The right extramural coronary artery samples: $1 \mathbf{P}=$ the proximal segment, $2 \mathbf{P}=$ the distal segment. The left extramural coronary artery samples: $1 \mathrm{~A}=$ the proximal segment, $2 \mathrm{~A}=$ the distal segment. The $1 \mathrm{~A}$ and $1 \mathrm{P}$ samples were taken $1 \mathrm{~cm}$ from the ostia. The $2 \mathrm{~A}$ samples were taken half-way between the coronary sulcus and the apex. The $2 \mathrm{P}$ samples were taken at the inferior margin more widespread plaques were noted with a single stenosis, in grade 3 atherosclerosis there was extensive plaque formation with stenosis in two or three arteries.

The hearts were immersion fixed in phosphate-buffered $4 \%$ formaldehyde. As shown in Figure 1, 2 samples were taken from each of the coronary arteries together with slices of the kidneys from all patients. The specimens were decalcified and embedded in paraffin, cut at 6-8 $\mu \mathrm{m}$ and stained with the Periodic Acid-Schiff (PAS) reaction to show glycoproteins, sirius red for connective tissue [4] and alcian blue $(\mathrm{pH}=2.6)$ for acid mucopolysaccharides. By means of an ocular micrometer the intimal thickness of all arterial samples was measured at the site of maximal plaque thickness as well as in the plaque-free area (when this was present).

We defined plaque as a local thickening of the tunica intima characterised by one or more of the following changes: accumulation of cholesterol, presence of lipophages, inflammation, vascular invasion, fibrosis and calcification. The plaque-free part was defined as lacking these changes but was, of course, the site of diffuse intimal thickening in the age group studied. Under the intimal areas thus defined the thickness of the tunica media was measured, and in these same tunica media areas the occurrence of connective tissue, PAS-positive and alcian-positive material was quantitated by pointcounting. We used a square ocular grid with 63 points at $400 \times$ magnification. Within each of the areas studied 5 random fields were counted. The average number of points counted in each area was 100. For each staining reaction the positive area-fraction was determined.

\section{Statistical analysis}

Student's t-test or Wilcoxon's test for non-paired comparison was used, and a $p$ value of less than 0.05 was considered as the limit of significance (two-tailed test) [5].

\section{Results}

Clinical (ischaemic) heart disease was equally frequent in both groups of patients. By grading the atherosclero- 


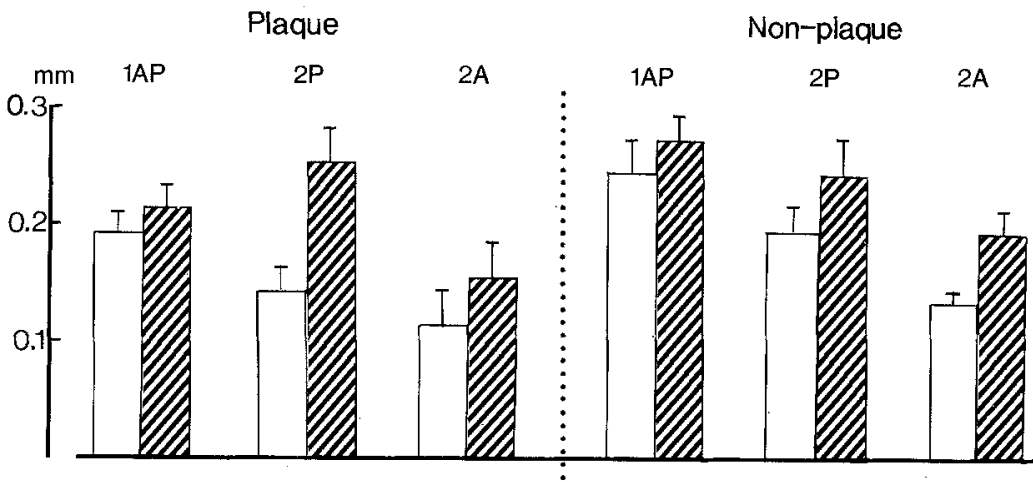

Fig. 2. The thickness of tunica media. $1 \mathrm{AP}=$ the average results from the proximal segment of the right and left extramural coronary artery. (Plaque, 2P: $2 p<0.02$. Non-plaque, $2 \mathrm{~A}: 2 p<0.005$, remaining segments not significant). $1 \mathrm{bar}=1 \mathrm{SEM} . \square$ Diabetic patients; non-diabetic subjects

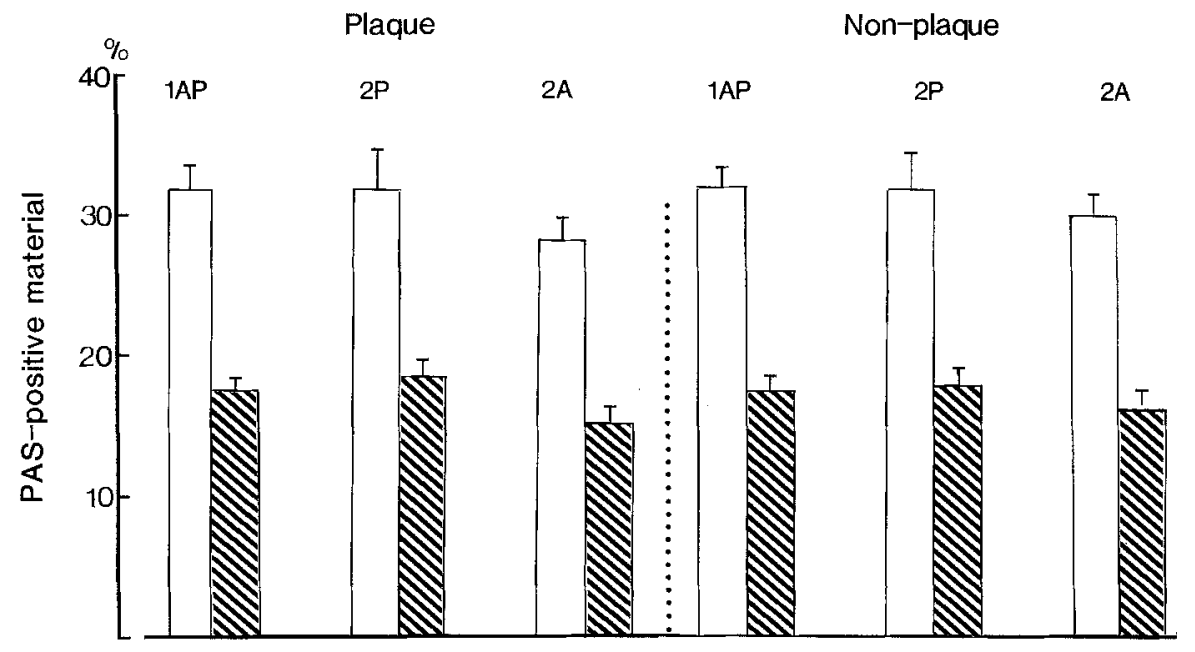

Fig. 3. The fractional amount of PAS-positive material in tunica media in the four segments obtained from the extramural coronary arteries. $1 \mathrm{AP}=$ the average results from the proximal segments of the arteries. (For all segments $2 p<0.01$ ). $1 \mathrm{bar}=1 \mathrm{SEM}$. $\square$ Diabetic patients; non-diabetic sub- sis semiquantitatively we found 4 patients in the group with diabetes with grade 2 and 6 patients with grade 3 atherosclerosis. Among the non-diabetic subjects 1 with grade 1, 4 with grade 2 and 5 with grade 3 atherosclerosis were found. The average heart weight among diabetic patients was $436 \mathrm{~g}(340-600)$; for the non-diabetic subjects it was $332 \mathrm{~g}(230-480)$; this difference was significant $(2 p<0.01)$. However, a similar difference was not noted in the body mass index (Table 1 ). There was no difference in the thickness of tunica intima between the two groups in plaque-free areas. Compared with this we found a 5-fold intimal thickening in plaque areas without difference between the two patient groups. Measurements of the tunica media thickness in the central samples (1AP) showed no difference either. However, in the peripheral segments $(2 \mathrm{~A}$ and 2P) with and without plaque the tunica media from diabetic patients was significantly thinner than in nondiabetic subjects (Fig. 2$)(2 p<0.02$ and $<0.005)$.

The PAS-positive material occurred diffusely between the muscle cells of the tunica media in both groups of patients. When point-counting was performed on the tunica media no difference was found in the amount of PAS-positive material under plaque and plaque-free tunica intima. However, the PAS-positive area fraction was significantly (i.e. about $50 \%$ ) increased in the tunica media of vessels from the diabetic patients as compared to non-diabetic subjects $(2 p<$ 0.01 ) (Fig. 3). The alcianophilic material was also found diffusely throughout the whole tunica media without difference between the tunica media below plaques and plaque-free intima in the two groups of patients. But, the alcianophilic area fraction was observed to be significantly (about 50\%) lower in the tunica media from diabetic patients as compared to nondiabetic subjects in all the analysed samples (Fig.4, $2 p<0.02$ ). It appears from Figures 3 and 4 that there was little variation in the fractional contents of each of these substances within each of the two groups of patients.

The amount of connective tissue (Sirius red) estimated in the proximal segment of the arteries was found to be the same in tunica media below tunica intima with plaque comparing diabetic and non-diabetic subjects (diabetic: $61.9 \pm 10.2$ vs non-diabetic: $55.3 \pm$ $9.4 \mathrm{X} \% \pm \mathrm{SD}$ ). However, in the tunica media outside intimal plaque the amount of connective tissue was significantly increased in arterial samples from diabetic patients (Fig. 5, $2 p<0.025$ ). In the peripheral part of the arteries no difference was seen in the content of connective tissue in the tunica media. In Figures 2-5 the results obtained from the central segments were pooled since they were identical and represent vessels of approximately equal calibre. The results of the pe- 


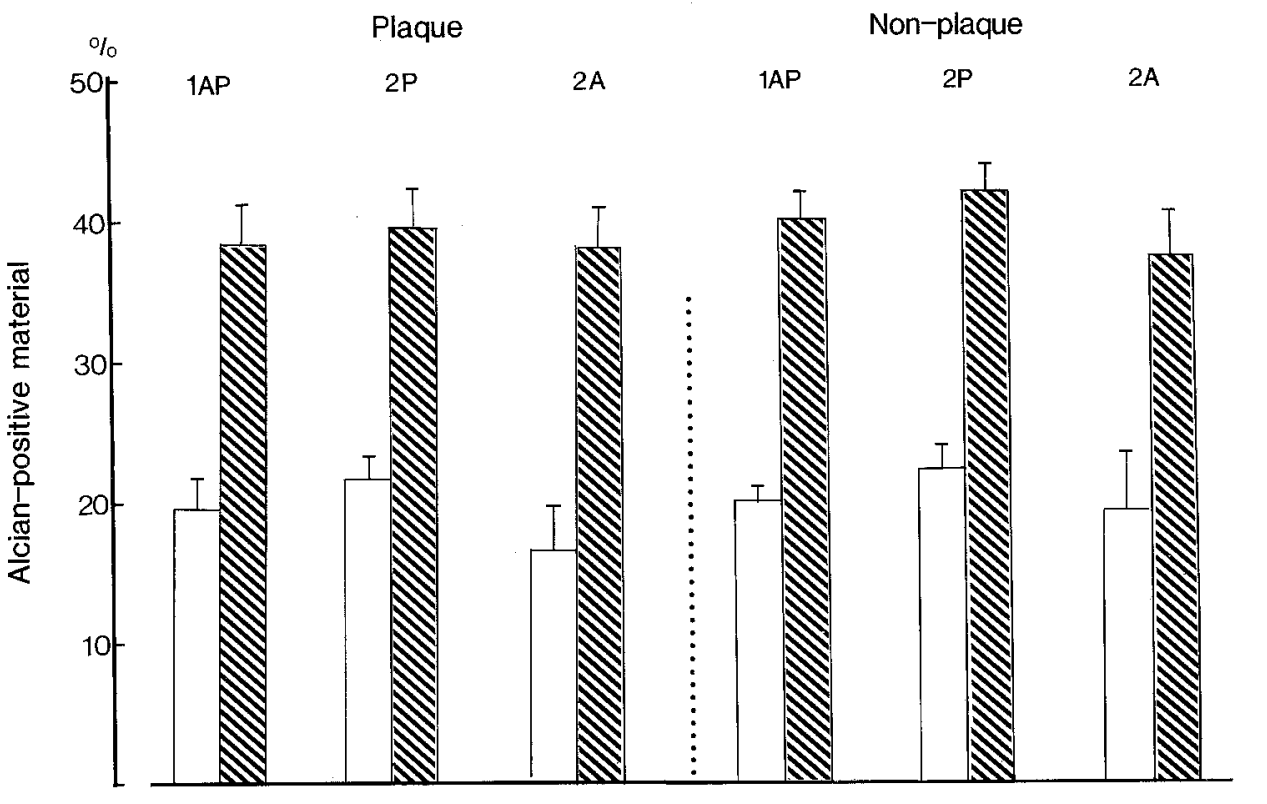

Fig.4. The fractional amount of alcianophilic material in tunica media in the four segments obtained from the extramural coronary arteries. $1 \mathrm{AP}=$ the average results from the proximal segments of the arteries. (For all segments $2 p<0.02) .1$ bar $=1$ SEM. $\square$ Diabetic patients; non-diabetic subjects

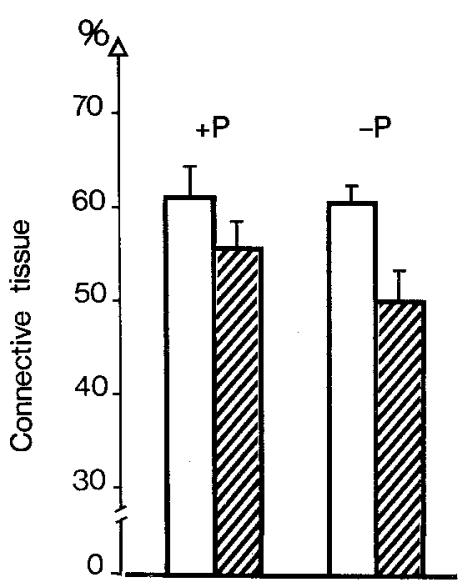

Fig.5. The fractional amount of connective tissue in tunica media in the proximal segments of the right and left extramural coronary arteries. $+\mathrm{P}=$ areas below tunica intima with plaque, $-\mathrm{P}=$ areas below plaque-free tunic intima. $1 \mathrm{bar}=1$ SEM. $\square$ Diabetic patients; non-diabetic subjects

ripheral segments - though also identical - are shown separately, since the left sample constantly had a smaller diameter than the right one.

No correlation was noted between the area fraction of PAS-positive material or alcianophilic material in the tunica media and the known duration of diabetes. Neither was there any relation to the heart weight. In the kidneys of the three uraemic diabetic patients severe chronic nephropathy with Kimmelstiel-Wilson lesions was found, in the remaining kidneys only unspecific changes were seen. But there was no difference in the fractional amount of PAS-positive material in the arterial tunica media between patients with Kimmelstiel-Wilson lesions and the remaining diabetic patients.

\section{Discussion}

Previously we have shown, using semiquantitative techniques, that PAS-positive material accumulated in the extramural coronary arteries from Type 2 diabetic patients [6]. However, in these studies no separation between areas with and without atherosclerotic plaques was made. In the present quantitative morphological study it clearly appeared that PAS-positive substance accumulated diffusely in the tunica media irrespective of atherosclerotic plaque location. In the same area of vessels obtained from diabetic patients the amounts of acid mucopolysaccarides evaluated by histochemical techniques were significantly reduced.

The nature of the accumulated PAS-positive substance in the tunica media is unknown. The arterial smooth muscle cells are surrounded by a PAS-positive basement membrane and recent culture studies have demonstrated that serum from Type 1 (insulin-dependent) and Type 2 diabetic patients enhanced the accumulation of basement membrane material and fibronectin [7]. In another in vitro study we have observed that growth hormone, which is increased in diabetic patients, has the same effect, but with a reduced content of acid mucopolysaccharides (glycosaminoglycans) in the basement membrane material $[7,8]$. Therefore it is possible that the increased amount of PAS-positive substance seen in the tunica media from vessels of diabetic patients may be due to accumulation of basement membrane material around the medial smooth muscle cells.

It is of considerable interest that the common histopathological denominator of diabetic microangiopathy has been the PAS-positive but acid mucopolysaccharide-negative deposits in the smaller blood vessels $[6,9$, 10]. The similarity between the observations in the present study and those seen in diabetic microangiopathy 
suggests that some of the abnormalities found in the large blood vessels may belong to the diabetic angiopathy.

It came to light that only the proximal part of the coronary arteries outside plaques contains more connective tissue (Sirius red) in the tunica media of diabetic patients than in non-diabetic subjects. It is possible that a similar difference had also appeared concerning the tunica media below plaques, if a larger number of hearts had been studied. Previously we were not able to demonstrate a difference in the amount of connective tissue evaluated by histological techniques between the coronary arteries from Type 2 diabetic patients, and non-diabetic subjects [6]. However, these earlier data were obtained with semiquantitative methods which may account for the discrepancy in results. In agreement with the present data are our previous in vitro experiments with cultured arterial smooth muscle cells analysed with biochemical techniques. We found increased production of Type 1 collagen after addition of serum from young diabetic patients [7].

The width of the tunica intima and the semiquantitative gradation of atherosclerosis were similar in diabetic patients and non-diabetic subjects. This observation - and the equal increase in the fractional amount of PAS-positive material in the media below and outside plaques - suggest that the altered composition of the tunica media in diabetic patients is not caused by atherosclerosis.

In the arteries from diabetic patients we noted a reduced thickness of the tunica media, in central as well as peripheral samples (Fig.2), but the difference was only significant in the peripheral samples, which may be imputed to the moderate number of hearts investigated.

Our present observation of the tunica media thickness may not necessarily be in contradistinction to the study of Goodale et al. [11] who found appreciably greater thickness of the wall of extramural coronary arteries of diabetic patients, since the quantification of this study was carried out using calibre measurements which may have included the entire vessel wall. Goodale et al. [11] found a higher frequency of heart disease among male diabetic patients after matching for age, sex and coronary wall thickness. The degree of lumen reduction was not measured by either Goodale et al. [11] or by us. But Waller et al. [12] found no difference in the degree of lumen reduction of extramural coronary arteries between large groups of Type 2 diabetic patients with and without clinical heart disease, and a group of non-diabetic subjects with heart disease. Consequently the studies of Waller et al. [12] and Goodale et al. [11] both suggest that factors other than a simple narrowing of lumen of the extramural coronary arteries may be involved in the development of clinical heart disease. Of course, diabetic microangiopathy and also diabetic cardiomyopathy may be involved. But additionally, the severe alterations of the tunica media shown in this study may have physiological consequences such as altered permeability and mechanical properties.

We found no relationship between the alterations observed and the duration of diabetes; which may be imputed to the well known difficulty in defining the onset of diabetes in patients with Type 2 diabetes mellitus.

It appears from the present quantitative histological report that certain abnormalities develop irrespective of atherosclerotic plaque location in the extramural coronary arteries from patients with Type 2 diabetes. The alterations observed share characteristics with those seen in diabetic microangiopathy. Therefore it seems reasonable to consider those phenomena to be part of the diabetic angiopathy and in particular part of a non-atherosclerotic diabetic macroangiopathy.

\section{References}

1. Bryfogle JW, Bradley RF (1957) The vascular complications of diabetes mellitus. A clinical study. Diabetes 6: 159-167

2. Ledet T, Gøtzsche O, Heickendorff L (1984) The pathology of diabetic cardiopathy: pathogenetic reflections. In: Jarrett J (ed) Diabetes and heart disease. Metabolic aspects of cardiovascular disease, Vol 2. Elsevier, Amsterdam New York Oxford, pp 25-46

3. Lundbaek K (1971) In: Lundbaek K, Keen H (ed) Blood vessel disease in diabetes mellitus. Acta Diabetol Lat 8 [Suppl 1]: 3

4. Sweat F, Puchtler H, Rosenthal SI (1964) The Sirius-red (F3BA) method as a stain for connective tusse. Arch Pathol 78: 69

5. Snedecor GA, Cochran WG (1972) Statistical methods. The Iowa University Press Ames, Iowa, USA

6. Ledet T (1968) Histological and histochemical changes in the coronary arteries of old diabetic patients. Diabetologia 4:268-272

7. Ledet T, Vuust J (1980) Arterial procollagen type I, type III, and fibronectin. Effects of diabetic serum, glucose, insulin, ketone, and growth hormone studied on rabbit aortic myomedial cell cultures. Diabetes 29: 964-970

8. Ledet T, Heickendorff L (1985) Growth hormone effect on accumulation of arterial basement membrane-like material studied on rabbit aortic myomedial cell cultures. Diabetologia 28: 922-927

9. Blumenthal HT, Axel M, Goldenberg S (1960) A study of lesions of the intramural coronary artery branches in diabetes mellitus. Arch Pathol 70: 13-28

10. Ledet T (1976) Diabetic cardiopathy. Quantitative histological studies of the heart from young juvenile diabetics. Acta Pathol Microbiol Scand [A] 84: 508-516

11. Goodale D, Daoud AS, Florentin R, Lee KT, Gittelsohn A (1962) Chemico-anatomic studies of arteriosclerosis and thrombosis in diabetics. I: Coronary arterial wall thickness, thrombosis and myocardial infarcts in autopsied North Americans. Exp Mol Pathol 1: 353-363

12. Waller BF, Palumbo PJ, Lie JT, Roberts WC (1980) Status of the coronary arteries at necropsy in diabetes mellitus with onset after age 30 years. Am J Med 69: 498-506

Received: 6 March 1987

and in revised form: 1 October 1987

Dr. H. Dybdahl

University Institute of Pathology

Århus Kommunehospital

DK-8000 Århus C

Denmark 